\title{
Steady Functioning of an Enterprise in the Conditions of Variable Economy
}

\author{
Andrey Vladimirovich Shmidt \\ South Ural State University, Candidate of Science (Economics), \\ Pro-rector for academic affairs, Chelyabinsk, Russia \\ Email: shmidtav@susu.ac.ru
}

\section{Tatyana Albertovna Khudyakova}

\author{
South Ural State University, Candidate of Science (Economics), \\ Head of Department "Economics and Service Manadgment", Chelyabinsk, Russia \\ Email: khudiakovata@susu.ac.ru
}

\section{Doi:10.5901/mjss.2015.v6n4p274}

\section{Abstract}

The article considers the modern theoretical and methodological approaches to the assessment of the economic sustainability of industrial enterprises. Development of long-term strategy aimed at achieving a set of socio-economic indicators, should include an analysis of the dynamics and ensure the minimization of cost for achieving the goal in the conditions of variable environment. The objectively existing and fundamentally fatal uncertainty in the external environment system causes disturbing impacts arising from its moments towards the goal, and determine formation of variable indicators at the output of the system. It is offered to make an assessment of the economic sustainability of the enterprise on the basis of probabilistic-statistical methods.

Keywords: economic sustainability, modeling of economic system, economic and mathematical models, industrial enterprise, sustainable economic development.

\section{Introduction}

Projecting methods to evaluate the industrial enterprise on criteria of economic stability in terms of dynamics is connected with the development of methodological approaches to form the system of its figures. At the present stage of the economic thought the figures providing for the evaluation of dynamic economic stability are of great importance for identification of reserves and possibilities to increase the efficacy of performance and development of mini-economic systems under the condition of uncertain environment with the high degree of variability of disturbing influences. Identifying figures of economic stability of a mini-economic entity completes the information derived from standard procedures of economic analysis (Ansoff, 1989).

Industrial enterprises discriminate the following components of the specified factor groups, disturbing and control influences: deterministic factors - cost of equipment, depreciation rates, different expenditure rates of material recourses and productivity; uncertain factors - cost of material resources, volume of sales, product demand, tax level etc. management decision factors - product price, wage rate, rate of marketing costs etc.

It's worth saying that these are the fluctuation of uncertain factors which are essentially incoming disturbing influences of a mini-economic system. To solve the task of steady performance can be reduced to finding methods compensating for the negative influence of the uncertain factors fluctuation.

To introduce a special quantitative index of economic stability this concept should be treated in terms of its dynamics.

\section{Theoretical Framework and Literature Review}

It is regular that the difficulty of constructing special algorithms for the analysis of mini-economic entities' stability under the condition of uncertain environment is connected with the level of organizational forms of modern enterprises (Bayev et al., 2001). The basic characteristics of modern enterprises as open social and economic systems are the complex organizational structure, instability and uncertainty of disturbing influences. 
An industrial enterprise as a mini-economic system is characterized by a great lot of elements and their interconnections, constant influence of external and internal factors of macro-, meso-, and micro-environments of different degrees of variability. Meanwhile the internal corporative processes are difficult to formalize due to organization complexity. Thus, there appears a necessity to develop non-parametric economic and mathematical methods for identification of the economic stability figures characterizing the economic situation in mini-economic system, which is observed at the given temporal interval.

While identifying the economic stability figures in terms of the analysis of enterprise operating results, it is reasonable to use the models bases on the analysis of cash flow.

In this case it is necessary to predict possible factor values and disturbing influences of different origin which affect the formation of cash flow, to analyze the quantitative influence of the disturbances of different level on the elements of cash flow structure determine the final figures of the enterprise operating results.

The combination of factors of different origin produces defined values of different figures (Khudyakova and Shmidt, 2006), characterizing the enterprise operating results: sales proceeds, taxable income, net transactional inflow and correspondent efficacy figures.

\section{Materials and Methods}

A possible interpretation of economic stability: let there be a trajectory of the development of a mini-economic system, reflecting changing parameters of its standing, and let there be some domain $\varepsilon^{*}$ of admissible deviation of a real phase trajectory of the set development trajectory. Then if there is a domain $\sigma^{\star}$ and if the real trajectory lying in the domain $\sigma^{*}$ never exceeds the domain $\varepsilon^{*}$ along all the trajectory, then a mini-economic dynamic system is steady. Such an approach is used for the analysis of technical systems stability by Lyapunov. Our interest lies in the dynamic economic stability and hereinafter making comparisons of economic systems stability we shall mean the very dynamic stability, since the temporal factor in this case is completely impossible to be excluded from consideration.

It seems that the principle moment in leaving the technical system in favor of the social-economical one is the fact that deviations of real development trajectories form an objective trajectory are of random and stochastical nature, and it's impossible to get exact information of these deviations. And if it is possible to judge the stability of a technical system by Lyapunov unequivocally, analyzing the difference equations, characterizing system's behavior, in terms of the analysis of economic stability due to the wide impossibility to propose a difference equation of the economic system performance, the conclusion of stability or instability can be drawn only with some possibility by means of the suggested methods. The crucial moment for identification of a stability index for the given class of systems is the property of deviation from real development trajectories which provides for the occurrence, though random, of these deviations, but it possible to determine the probability of a certain deviation of a real phase trajectory from an objective trajectory at every point, so there appears to be a law of frequency distribution of these deviations. The existence of the law of frequency distribution of deviations of a real phase trajectory of a mini-economic system from an objective trajectory makes it possible to determine the probability of deviations of a real phase trajectory from an objective trajectory, with the set objective anyway achieved. The possibility, that a real phase trajectory of performance and development of a mini-economic system will not exceed the objective domain (domain $\varepsilon^{\star}$ ) along all the objective trajectory, is an index of functional stability of an object-oriented a social-economic system against the object view. In this respect the task to analyze the economic stability against the object view is to identify the possibility degree for the system to achieve the object view under the condition of uncertain parameters of the external environment.

If the stability of system performance is the probability of achieving some quantitatively characterized objective, the stability analysis task is reduced to finding the law of frequency distribution of quantitative results of mini-economic system performance and finding the probability of objective achievement using this law.

The possible approach for the solution of the task of steady mini-economic system performance of an industrial enterprise against the set object view is to determine the function of distributing objective functional by means of the laws of joint distribution of random system parameters. In this respect the task to determine the laws of distribution of the objective function of a random value can be used to determine the probability of the given function to enter its acceptable region (Ventsel and Ovcharov, 2000, Bolshakov, 1983). Consider an example when the objective functional of minieconomic system is a function of random parameters $x 1, x 2, \ldots, x n$.

$Z=\varphi\left(x_{1}, x_{2}, \ldots, x_{n}\right)$,

when limited 


$$
Z \geq q^{* *}\left(x_{1}, x_{2}, \ldots, x_{n}\right) .
$$

The probability of steady performance of a mini-economic system against the object view can be determined with the distribution function $R(\mathrm{Z})$.

$$
R(Z)=P\left\{Z \geq q^{* *}\right\} \text {. }
$$

Consider the objective function depending on the combination of laws of distributing independent system parameters, distributed according to the normal law and can be interpreted as

$$
Z=\sum_{i=1}^{n} a_{i} x_{i}+b
$$

where ai and $b$ - deterministic coefficients; $x_{i}$ - random parameters of a mini-economic system.

Consider an example when the objective function is a sum of random values $x i$ with distribution densities $f\left(x_{i}\right)$ respectively. Find the distribution density of the probabilities $f(Z)$ of a stochastical value ${ }^{z=\sum_{i=1}^{n} a_{i} x_{i}+b}$ Figure 1 clarifies the set task.

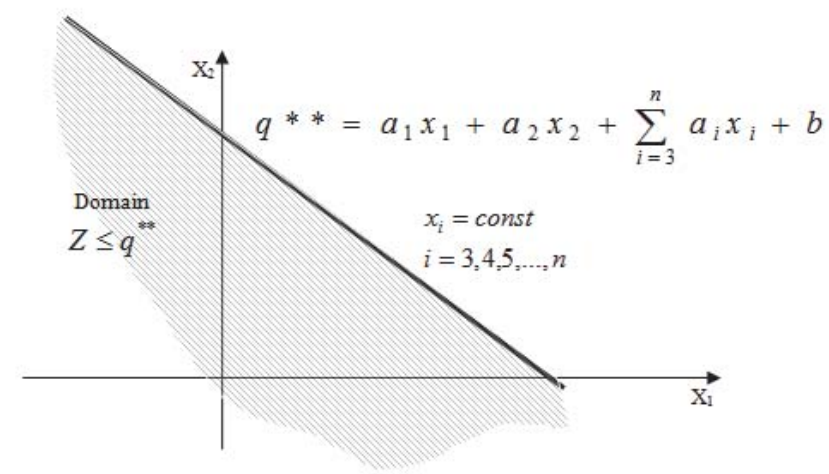

Figure 1. Plotting the domain $Z=\sum_{i=1}^{n} a_{i} x_{i}+b \geq q^{* *}$

Distribution function of a random value $Z$ probabilities

$$
F(Z)=P\left(Z \geq q^{* *}\right)=P\left(\sum_{i=1}^{n} a_{i} x_{i}+b \geq q^{* *}\right)
$$

can be achieved by integrating the tuple probability density $f\left(\sum_{i=1}^{n} a_{i} x_{i}+b\right)$ in the domain, situated above the line $a_{1} x_{1}+a_{2} x_{2}+\sum_{i=3}^{n} a_{i} x_{i}+b=q^{* *}$

. Here we take $q^{* *}$ as a deterministic value. For every fixed value $x i$ the value $x$ must provide for the fulfillment of the condition $-\infty<x<Z-x_{i}$. Then it is possible to get (Ventsel \& Ovcharov, 2000, Bolshakov, 1983):

$$
F(Z)=\int_{-\infty}^{\infty} \int_{-x_{i}}^{\infty} f\left(x_{1}, x_{2}, \ldots, x_{n}\right) d x_{1} d x_{2} \ldots d x_{n}
$$

In particular if $x i$ and $x j$ are statistically independent, the common distribution density of probabilities can be interpreted as a factum of cofactors and the formula (6) appears as

$$
F(Z)=\int_{-\infty}^{\infty} \int_{-x_{i}}^{\infty} f\left(x_{1}\right), f\left(x_{2}\right), \ldots, f\left(x_{n}\right) d x_{1} d x_{2} \ldots d x_{n}
$$

The distribution density of random value probabilities $Z=\sum_{i=1}^{n} a_{i} x_{i}+b \geq q^{* *}$ found, through differentiation of $F(Z)$ along $Z$. 
In terms of the Leibnitz-Newton theorem the running integral with the running limit inferior is the continuous function and the derivative from this integral over the variable is the primitive of this integral (Cooper \& McGillem, 1989).

As the variable $Z$ appears only in the limit inferior of the integrals. Thus, it is worth saying that in this case $f(Z)$ is a convolution of one-dimensional distribution densities of random values probabilities.

Then it is possible to write

$$
f(Z)=\frac{d F(Z)}{d Z}=\int_{-\infty}^{\infty} f\left(x_{i}-Z\right) \int f\left(x_{i+1}\right) \ldots d x_{1} \ldots d x_{n-1}
$$

It is worth saying that the convolution of the (8) type can be attributed to any factor of the mini-economic system xi.

Taking into consideration that linear conversions of the laws of density distribution of a mini-economic system environment do not lead to changing the law of distribution density of an objective functional, the condition of the objective function to enter the set parameters can be interpreted as $q^{* *} \leq Z \leq q^{*}$

$$
P\left(q^{* *} \leq Z \leq q^{*}\right)=\int_{q^{*}}^{q^{*}} f(Z) d Z=\Phi\left(\frac{q^{*}-\bar{Z}}{\sigma_{Z}}\right)-\Phi\left(\frac{q^{* *}-\bar{Z}}{\sigma_{Z}}\right)
$$

where $\Phi($.$) - erf integral (Laplace's function).$

As an example, consider the law of distributing an objective functional of a mini-economic system depending on the distribution of three random values of environmental factors. The function is set as

$$
Z=a_{0} x_{0} x_{1}+a_{2} x_{2}+b \text {, }
$$

where $Z$ - annual balance profit, $x 0$ - enterprise sales volume, $x 1$ - price for an item of product, $x 2$ - conditionally variable costs, $a 1, a 2<0$ - regular coefficients, $b$ - conditionally regular costs. To simplify the solution reduce the task to two random parameters and the formula (10) will be interpreted as

$$
Z=a_{1} x_{1}+a_{2} x_{2}+b
$$

where ${ }^{a_{1}=a_{0} x_{0}}$. Random parameter $x 0$ taken through the solution of three tasks: the first solution is achieved with $\left(x_{0}-3 \sigma_{x_{0}}\right)$, the second $-x_{0}$ and the third $\left(x_{0}+3 \sigma_{x_{0}}\right)$. There are the intervals for the solution $\pm 3 \sigma_{x_{0}}$ (where $\sigma_{x_{0}}$ - mean-square deviation of the factor ${ }^{x_{0}}$ ) of the mathematical expectation ${ }^{x_{0}}$, as the probability with such deviations of a variable is insignificant. With the received probability of steady performance it is possible to plot a diagram $R=\varphi(x 0)$ as it is shown in the figure 2, and to evaluate the influence of the variable ${ }^{x_{0}}$ on the probability of steady performance of a mini-economic system.

As the common distribution density of statistically independent values $x 1$ and $x 2$ can be interpreted as (Ventsel and Ovcharov, 2000, Bolshakov, 1983)

$$
f\left(x_{1}, x_{2}\right)=f\left(x_{1}\right) f\left(x_{2}\right)=\frac{1}{\sqrt{2 \pi} \sigma_{x_{1}}} e^{-\frac{\left(x_{1}-\bar{x}_{1}\right)^{2}}{2 \sigma_{x_{1}}{ }^{2}}} \frac{1}{\sqrt{2 \pi} \sigma_{x_{2}}} e^{-\frac{\left(x_{2}-\bar{x}_{2}\right)^{2}}{2 \sigma_{x_{2}}{ }^{2}}},
$$

find the distribution density of the objective functional of a mini-economic system.

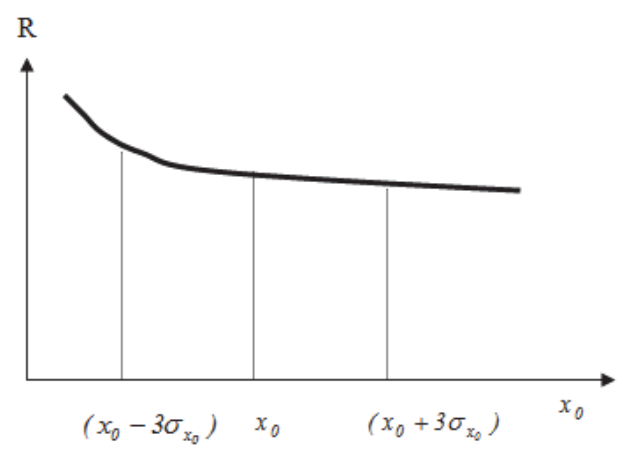

Figure 2. Dependence of the probability of steady performance of a mini-economic system at the time moment $t i$ of the stochastic factor $x 0$ 
The function of distributing probabilities of the random value $Z$

$$
F(Z)=P\left(Z \geq q^{* *}\right)=P\left(a_{1} x_{1}+a_{2} x_{2}+b \geq q^{* *}\right) \text { can be derived through integrating a two-dimensional distribution }
$$

density (12) in the domain situated above the line $q^{* *}=a_{1} x_{1}+a_{2} x_{2}+b$. For example, the limit in the formula

$x_{1}=\frac{y-a_{2} x_{2}-b}{a_{1}}$

Differentiating the formula with $d Z$ gives the distribution density of random value $Z$ probabilities like in (Kapur and Lamberson, 1980):

$$
\begin{aligned}
& f(Z)=\int_{-\infty}^{\infty} \frac{\operatorname{sign}\left(a_{1}\right)}{2 \pi \sigma_{1} \sigma_{2}} e^{-\frac{1}{2}\left[\frac{\left(\frac{Z-a_{2} x_{2}-b}{a_{1}-\bar{x}_{1}}\right)^{2}}{\sigma_{x_{1}}^{2}}+\frac{\left(x_{2}-x_{2}^{2}\right.}{\sigma_{x_{2}}^{2}}\right]} d x_{2}=\frac{\operatorname{sign}\left(a_{1}\right)}{2 \pi \sigma_{1} \sigma_{2}} \int_{-\infty}^{\infty} e^{-A x_{2}^{2}+2 B x_{2}+C} d x_{2}, \\
& \text { Where } A=\frac{1}{2 a_{1}^{2}}\left[\frac{a_{2}^{2}}{\sigma_{x_{1}}^{2}}+\frac{a_{1}^{2}}{\sigma_{x_{2}}^{2}}\right] ; \\
& B=-\left[\frac{Z-b-a_{1} \bar{x}_{1}}{a_{1}^{2} \sigma_{x_{1}}^{2}}-\frac{Z-b-a_{1} \bar{x}_{1}+a_{2} \bar{x}_{2}}{a_{1} \sigma_{x_{1}} \sigma_{x_{2}}}-\frac{\bar{x}_{2}}{\sigma_{x_{2}}^{2}}\right] ; \\
& C=-\frac{1}{2}\left[\frac{\left(y-b-a_{1} x_{1}\right)^{2}}{a_{1}^{2} \sigma_{I}^{2}}+\frac{\bar{x}_{2}^{2}}{\sigma_{x_{2}}^{2}}\right] .
\end{aligned}
$$

If the latter expressions are integrated into the distribution density $f(Z)$ formula the result is

$$
f(Z)=\frac{\operatorname{sign}\left(a_{1}\right) \sqrt{\pi}}{2 \pi \sigma_{x_{1}} \sigma_{x_{2}} \sqrt{A}} e^{-\frac{A C-B^{2}}{2}} \text {. After conversion } f(Z)=\frac{1}{\sigma_{Z} \sqrt{2 \pi}} e^{-\frac{(Z-\bar{Z})^{2}}{2 \sigma_{Z}^{2}}} \text {, where sign(a1); } \bar{Z}=a_{1} \bar{x}_{1}+a_{2} \bar{x}_{2}+b
$$

mathematical expectation of an objective function; $\sigma_{Z}^{2}=D[Z]$ - dispersion of an objective function.

Now knowing the law of density distribution of a random value of the enterprise objective functional, it is sufficient to determine the probability of steady performance of a mini-economic system with the expression

$$
R=P\left(q^{* *} \leq Z \leq q^{*}\right)=\int_{q^{*}}^{q^{*}} \frac{1}{\sigma_{Z} \sqrt{2 \pi}} e^{-\left(\frac{Z-\bar{Z}}{2 \sigma_{z}^{2}}\right)^{2}} d z \text {. }
$$

After these procedures the formula (13) can be expressed as

$$
R=\Phi\left(-\frac{q^{*}-\bar{Z}}{\sigma_{Z}}\right)-\Phi\left(-\frac{q^{* *}-\bar{Z}}{\sigma_{Z}}\right) \text {. }
$$

\section{Conclusion}

Thus, the economic stability of an industrial enterprise is its ability to achieve the performance and development objectives within certain time-limits. A sign of the enterprise stability is the entrance of the enterprise cost figures into the target domain and the quantitative sign of the economic stability of an enterprise is the probability to meet the objective within certain time-limits.

The category of the enterprise economic stability against the objective is more informative rather than stability as an ability of a system to return to the balance state under external disturbing circumstances. The probability to achieve the objective within certain time-limits can span from 0 to 1 while the ability of a system to return to the start state can be estimated only by two values: a system is either able to return to the start state or not.

The evaluation of the enterprise economic stability should rest on a systematic approach for the usage of complex systems' stability principles. This study suggests the basic principles of the systematic approach for examining economic stability of an enterprise. It appears that stability is an external demonstration of internal properties of the very object.

The analysis of the enterprise stability is made on the basis of the cash flow generated by the enterprise, internal and external disturbing influences, limits of the target domain. The study offers a classification of disturbances influencing the dynamics of an enterprise and correspondently the dynamics of its cash flow.

There have been developed mathematic models of predicting changes of the cash flow generated by an enterprise under the influence of disturbances and evaluating a random event of the entrance of the enterprise cost figures into the target domain. The models allow determining the dependence of the enterprise stability on its "starting" state, creating the 
necessary basis for the stability control, to evaluate the efficacy of this control in terms of comparing the stability increasing expenditures with the stability index.

\section{Acknowledgements}

This publication was prepared within the framework of a research project №15-32-01051 supported by the RHF.

\section{References}

Ansoff, I. (1989). The Strategic Management. Moscow: Ekonomika Publishers.

Bayev, I.A., Shiryayev, V.I., Shiryayev, E.V. (2001). The Dynamic Theory of Form: Monograph. Chelyabinsk: SUSU Publishers.

Bayev, I.A., Shmidt, A.V. (2012). Management of economic stability of the enterprise in the context of increasing its competitiveness. Bulletin of UrFU. Series: Economics and management, 3, 50-63.

Bolshakov, V.D. (1983). The Theory of Observational Errors. Moscow: Nedra Publishers.

Cooper, J., McGillem, M. (1989). Probabilistic Methods of Analyzing Signals and Systems. Moscow: Mir Publishers.

Kapur, K., Lamberson, L. (1980). Reliability of System Engineering. Moscow: Mir Publishers.

Khudyakova, T.A., Shmidt, A.V. (2006). Research, Evaluation and Prediction of Economic Stability of an Industrial Enterprise. Chelyabinsk: SUSU Publishers.

Khudyakova, T.A. (2014). The role of controlling during managing sustainability of industrial enterprises Innovative development of modern science: collection of articles of the International scientific-practical conference, 293-295.

Shmidt, A.V. (2011). The Genesis of the concept of «economic stability» industrial enterprises. Business. Education. Right. Newsletter of Volgograd Institute of business, 4, 20-30.

Shmidt, A.V. (2011). The essence and the indicators of economic sustainability of the industrial enterprises. Business. Education. Right. Newsletter of Volgograd Institute of business, 3, 55-66.

Shmidt, A.V., Khudyakova, T.A. (2013). Modelling of management of development of industrial enterprises by economic criteria of the stability. Socio-economic problems of the modern Russian economy: monograph (250-282). Moscow: Economic newspaper.

Smornov, N.V., Dudin-Barkovskiy, I.V. (1965). A Course of the Probability Theory and Mathematical Statistics for Engineering Applications. Moscow: Nauka Publishers.

Ventsel, E.S., Ovcharov, L.A. (2000). The Theory of Probability and Its Engineering Applications. Moscow: Vysshaya Shkola Publishers.

Ventsel, E.S., Ovcharov, L.A. (2000). The Theory of Random Processes and Its Engineering Applications. Moscow: Vysshaya Shkola Publishers. 